\title{
DELAY IN THE DIAGNOSIS OF INVASIVE DUCTAL CARCINOMA DUE TO AN INFECTIOUS MASTITIS: CASE REPORT
}

Thiago Martins de Abreu', Arthur Gomes Pidde1, Pedro Henrique de Ávila Perillo', Silvaleide Ataides Assunção", Ianca Leandra Santos², Débora Sara de Almeida Cardoso ${ }^{3}$

1 Universidade Federal de Goiás - Goiânia (GO), Brazil.

${ }^{2}$ Faculdade Unida de Campinas - Goiânia (GO), Brazil.

${ }^{3}$ Centro Avançado de Diagnóstico da Mama, Hospital das Clínicas, Universidade Federal de Goiás - Goiânia (GO), Brazil.

Introduction: Breast cancer is the most common cause among women, with invasive ductal carcinoma (ICD) being the most prevalent and having great phenotypic and genotypic heterogeneity and the ability to metastasize. In turn, mastitis is an inflammation in the breast region, infectious or noninfectious causes, most commonly affecting lactating women. Objectives: It is intended to report a case of ICD accompanied by infectious mastitis and its reverberations. Case Report: A 27-year-old woman presents with a clinical history of mastitis in her left breast that occurred about 3 months ago after weaning her son. She also explained about the use of antibiotic therapy, multiple previous drainages, and local complication with engorgement, edema, erythema, and purulent drainage in the surgical ostium of the left breast. A new drainage and material collection was carried out. Computed tomography of the chest indicated a solid heterogeneous lesion, dense, irregular contours, with areas of air trapping, liquefaction, and cleavage plane with the left pectoralis major muscle and ipsilateral axillary adenomegaly. Pathological pathology confirmed the hypothesis of neoplasia, indicating grade III ICD in comedonecrosis. Immunohistochemistry demonstrated triple-negative character and culture, positivity for Corynebacterium renale. She started neoadjuvant chemotherapy with reduced breast volume and absence of secretion today. Discussion: This is a common and problematic situation in health systems. Repeated outpatient referrals, invasive processes without resolution, aggravating the patient's case. Moreover, it is noted that mastitis has delayed the diagnosis of the neoplasm, which, depending on the delay, may result in a worse prognosis or a more aggressive or expensive treatment. Conclusion: The diagnostic investigation of neoplasms is of great importance in case of prolonged mastitis not responsive to treatment, due to the fact that many mastitides may come from neoplastic processes that generate the lesion, which can make this injured area conducive to bacterial proliferation.

Keywords: Invasive Ductal Carcinoma; Mastitis; Breast Cancer. 